\title{
NOTE
}

\section{Prey size refugia and trophic cascades in marine reserves}

\author{
Marissa L. Baskett* \\ Department of Ecology and Evolutionary Biology, Princeton University, Princeton, New Jersey 08544, USA \\ Present address: National Center for Ecological Analysis and Synthesis, 735 State St., Suite 320, Santa Barbara, \\ California 93101, USA
}

\begin{abstract}
After the establishment of marine reserves, trophic cascades, with an increase in top predators, decrease in herbivores and increase in producers, are often expected but not consistently observed. Recent empirical results suggest that the lack of cascades in a Caribbean coral reef reserve may be due to larger herbivores escaping predation. To explore the potential for such prey size refugia to prevent trophic cascades after reserve establishment, I construct a simple trophic model with and without herbivore size refugia and determine the conditions necessary for herbivorous fish to decrease after the elimination of harvest mortality. Generally, cascades do not occur and herbivores increase if the effect of harvest on herbivores before reserve establishment is greater than the effect of predation after reserve establishment. The parameter space where herbivores increase is much greater when accounting for size refugia. The potential for prey size refugia to prevent cascades makes it an important dynamic to consider in community-level approaches to reserve design and monitoring.
\end{abstract}

KEY WORDS: Marine reserves $\cdot$ Trophic cascades $\cdot$ Size refugia $\cdot$ Predation $\cdot$ Model

\section{INTRODUCTION}

Trophic cascades occur frequently in coastal, hardsubstratum marine ecosystems (Pinnegar et al. 2000). Cascading effects often result from fisheries, as these tend to target top predators (Pauly et al. 1998), whose subsequent decline can lead to an increase in herbivores and decrease in producers. Therefore, as marine reserves, or no-take zones, are established to protect biodiversity and ecosystem structure (Allison et al. 1998), there is an expectation that predators will increase after reserve establishment, leading to cascades with herbivores decreasing and producers increasing. Accordingly, large-scale simulations of marine communities predict that trophic cascades are likely to occur in marine reserves (Walters 2000).

However, a meta-analysis of empirical studies of marine reserves failed to find evidence for consistently recurring trophic cascades (Halpern 2003). This has many possible explanations, such as cascades being restricted to a subset of a given marine community, variable recruitment, the influence of pathogens and food web complexity (Polis \& Strong 1996, Pinnegar et al. 2000, Halpern 2003). In a study of a Caribbean coral reef marine reserve, where larger herbivores increased along with piscivores after reserve establishment, Mumby et al. (2006) suggest that the reason for the lack of trophic cascades is the existence of prey size refugia, i.e. larger herbivores escape predation from piscivorous fish. In theoretical support of the potential for size refugia to prevent cascades, one model indicates that prey size refugia reduce the effects of top-down predator control in highproductivity environments (Chase 1999).

Trophic cascades after reserve establishment may not be a desirable outcome in the case of marine 
ecosystems such as Caribbean coral reefs, where a decrease in herbivores could increase the potential for algal overgrowth of corals after disturbances such as hurricanes (Mumby 2006). Therefore, predicting whether trophic cascades will occur is important to deciding whether marine reserves are an appropriate management strategy, as well as determining appropriate expectations for reserve monitoring. To assess the extent to which prey size refugia may prevent trophic cascades after reserve establishment, I explore a simple resource-based trophic model with and without prey size refugia.

\section{METHODS}

The model below takes the most basic form possible for generality and analytical tractability; this approach allows determination of the potential importance of incorporating size refugia in more detailed studies across marine ecosystems. In the model, the resource $R$ (e.g. algae) has input $R_{\mathrm{IN}}$ and flow rate $D$, analogous to classic chemostat models. Herbivores consume the resource at rate $\delta_{\mathrm{H}}$, which is converted into reproduction with efficiency $\beta_{\mathrm{H}}$. Herbivores are divided into 2 size classes, $H_{\mathrm{S}}$ and $H_{\mathrm{L}}$, where piscivores prey on herbivores in the smaller size class $H_{\mathrm{S}}$ at rate $\delta_{\mathrm{P}}$ and herbivores grow into the larger size class $H_{\mathrm{L}}$ at rate $\gamma$. Regardless of size, herbivores experience natural mortality at rate $\mu_{\mathrm{h}}$ and-before reserve establishmentconstant effort harvest mortality at rate $h_{\mathrm{H}}$. Piscivores $P$ convert predation on herbivores into reproduction with efficiency $\beta_{\mathrm{P}}$, experience natural mortality at rate $\mu_{\mathrm{P}}$, and experience harvest mortality at rate $h_{\mathrm{P}}$ before reserve establishment. Given these parameters, the dynamics are (Fig. 1):

$$
\begin{gathered}
\frac{\mathrm{d} R}{\mathrm{~d} t}=D\left(R_{\mathrm{IN}}-R\right)-\delta_{\mathrm{H}}\left(H_{\mathrm{S}}+H_{\mathrm{L}}\right) R \\
\frac{\mathrm{d} H_{\mathrm{S}}}{\mathrm{d} t}=\beta_{\mathrm{H}} \delta_{\mathrm{H}}\left(H_{\mathrm{S}}+H_{\mathrm{L}}\right) R-\left(\gamma+\mu_{\mathrm{H}}+h_{\mathrm{H}}+\delta_{\mathrm{P}} P\right) H_{\mathrm{S}} \\
\frac{\mathrm{d} H_{\mathrm{L}}}{\mathrm{d} t}=\gamma H_{\mathrm{S}}-\left(\mu_{\mathrm{H}}+h_{\mathrm{H}}\right) H_{\mathrm{L}} \\
\frac{\mathrm{d} P}{\mathrm{~d} t}=\left[\beta_{\mathrm{P}} \delta_{\mathrm{P}} H_{\mathrm{S}}-\left(\mu_{\mathrm{P}}+h_{\mathrm{P}}\right)\right] P
\end{gathered}
$$

The equivalent model without prey size refugia can be found by letting the herbivore growth rate into the larger size class $\gamma=0$. I take this approach-with Type I (linear) functional response for the predation dynamics, closed herbivore and predator dynamics, and resource-independent growth - for mathematical simplicity. More biologically realistic versions of this model warrant future study.

The effect of eliminating harvest mortality $\left(h_{\mathrm{H}}, h_{\mathrm{P}} \rightarrow 0\right)$ on the equilibrium values approximates the effect of

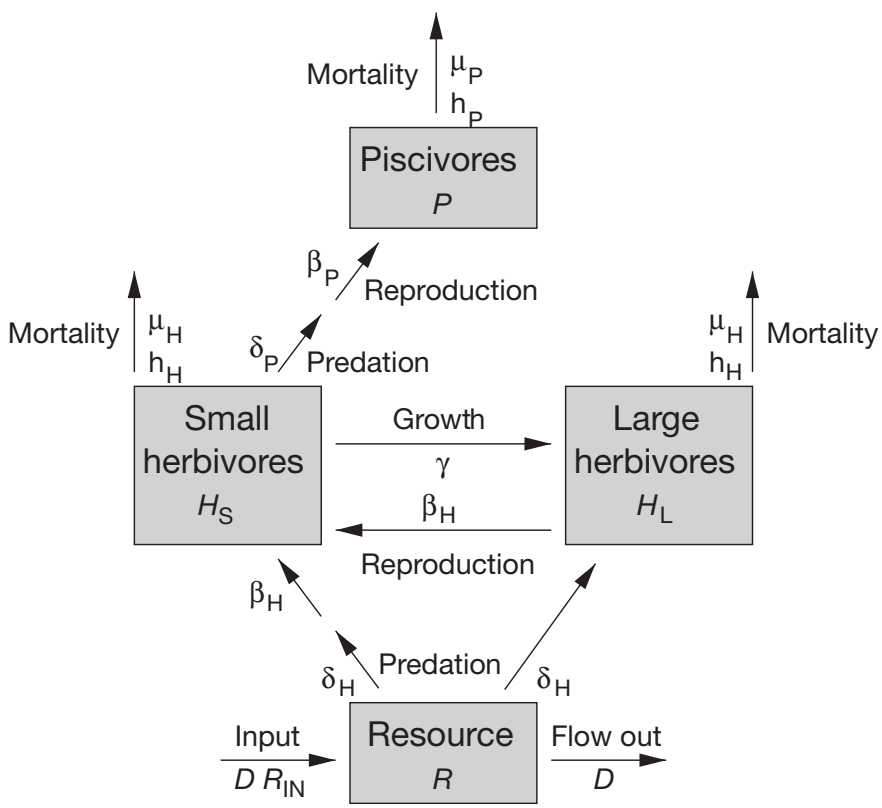

Fig. 1. Outline of the size refugia model (Eqs. 1 to 4 )

reserve establishment on expected population sizes. Specifically, whether the expected herbivore population decreases with the elimination of harvest mortality indicates whether trophic cascades occur after reserve establishment. The 'expected' population size is the locally stable equilibrium population size, given a set of parameters, as determined by the Routh-Hurwitz criteria for the Jacobian matrix analyzed at the equilibrium; see Appendix 1 for local stability analysis.

\section{RESULTS}

\section{Basic model}

As shown in Appendix 1, possible equilibria are with (1) the resource present and no herbivores or piscivores, (2) herbivores and the resource present and no piscivores, and (3) piscivores, herbivores and the resource all present (Fig. 2). Only 1 equilibrium is locally stable for a given set of parameters. I assume that the reproduction, growth, predation and natural mortality parameters have values such that the equilibrium with all 3 types of species present is the locally stable equilibrium when there is no harvesting. In other words, the herbivore population size in the equilibrium with the resource, herbivores and piscivores all present and with $h_{\mathrm{H}}=h_{\mathrm{P}}=0$ is the expected herbivore population size after reserve establishment. The locally stable equilibrium with $h_{\mathrm{H}}, h_{\mathrm{P}}>0$, and therefore the expected herbivore density before reserve establishment, depends on the harvest mortality values. 


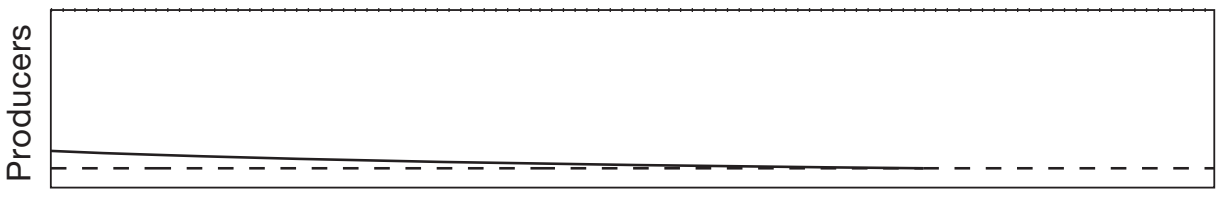

Fig. 2. Possible equilibria for the size refugia model as a function of the rate herbivores grow from the small to large size class (growth rate $\gamma)$. Dotted lines: equilibrium with the resource but no herbivores or piscivores; dashed lines: equilibrium with the resource and herbivores but no piscivores; solid lines: equilibrium with all 3 types of species. Only one equilibrium is locally stable for a given set of parameter values. Note that the equilibrium with all 3 types does not exist at high growth rates because the herbivores spend too little time in the smaller (available) size class to support the piscivores
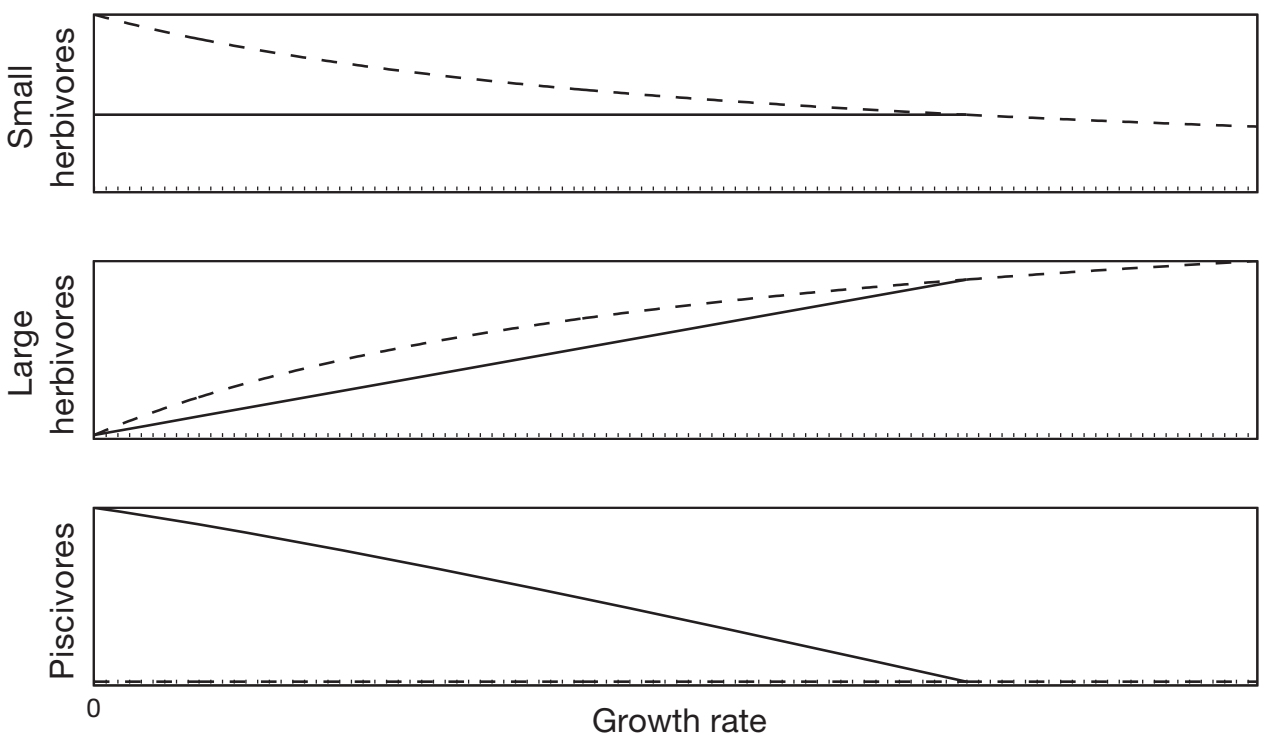

Below are the criteria for local stability of each equilibrium as well as the relevant criteria for herbivore increase (no trophic cascades), with and without size refugia, given the expected herbivore population size after reserve establishment.

To simplify the presentation of the model analysis, let $b_{\mathrm{H}}$ be the maximum herbivore birth rate:

$$
b_{\mathrm{H}}=\beta_{\mathrm{H}} \delta_{\mathrm{H}} R_{\mathrm{IN}}
$$

and $b_{\mathrm{P}}$ be the modified piscivore birth rate:

$$
b_{\mathrm{P}}=\beta_{\mathrm{P}} \delta_{\mathrm{P}} D / \delta_{\mathrm{H}}
$$

First, when $h_{\mathrm{H}}>h^{*}{ }_{\mathrm{H}, \mathrm{dep}}$, where:

$$
h^{*}{ }_{\mathrm{H}, \mathrm{dep}}=b_{\mathrm{H}}-\mu_{\mathrm{H}}
$$

the equilibrium without herbivores or piscivores is the locally stable equilibrium before reserve establishment. The above value can be interpreted as the herbivore harvest mortality being greater than the herbivore population growth rate (births minus deaths) or that the herbivores are being fished to depletion. As the predicted herbivore population size before reserve establishment tends to zero, and given the assumption of positive herbivore density after reserve establishment, herbivores always increase after reserve establishment in this case. This result is independent of the herbivore growth rate into the larger size class $(\gamma)$ and therefore it is the same with and without prey size refugia.
Second, when $h_{\mathrm{H}}<h^{*}{ }_{\mathrm{H}, \mathrm{dep}}$, the opposite of the stability criterion for the equilibrium without herbivores or piscivores, and $h_{\mathrm{P}}>h^{*}{ }_{\mathrm{P}, \mathrm{dep}, \mathrm{SR}}$, where:

$$
h_{\mathrm{P}, \mathrm{dep}, \mathrm{SR}}^{*}=\frac{b_{\mathrm{P}}\left[b_{\mathrm{H}}-\left(\mu_{\mathrm{H}}+h_{\mathrm{H}}\right)\right]}{\mu_{\mathrm{H}}+h_{\mathrm{H}}+\gamma}-\mu_{\mathrm{P}}
$$

the equilibrium with the resource and herbivores but without piscivores exists biologically and is locally stable. The above value can be interpreted as the piscivore harvest mortality being greater than the piscivore birth rate, given the herbivore population growth rate and growth into the unavailable size class, minus their natural mortality, or the piscivores are being fished to depletion. Note that this criterion depends on the herbivore harvest rate as well as the piscivore harvest rate because of the potential to harvest herbivores at a rate such that they can persist but with a population too small to support piscivores, i.e. ecological overfishing. When these criteria hold, herbivores will increase after reserve establishment (i.e. the herbivore density in the equilibrium with all 3 types of species and without harvesting is greater than the herbivore density in the equilibrium without piscivores and with harvesting) if

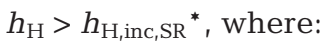

$$
h^{*}{ }_{\mathrm{H}, \mathrm{inc}, \mathrm{SR}}=\frac{b_{\mathrm{P}} b_{\mathrm{H}} \mu_{\mathrm{H}}}{b_{\mathrm{P}} \mu_{\mathrm{H}}+\mu_{\mathrm{P}}\left(\mu_{\mathrm{H}}+\gamma\right)}-\mu_{\mathrm{H}}
$$

or the effect of harvesting on herbivores before reserve establishment is greater than the effect of predation 
after reserve establishment. Without size refugia $(\gamma=0)$, the local stability criterion for the equilibrium without piscivores is $h_{\mathrm{P}}>h^{*}{ }_{\mathrm{P}, \mathrm{dep}, \mathrm{NSR}}$, where:

$$
h_{\mathrm{P}, \mathrm{dep}, \mathrm{NSR}}^{*}=\frac{b_{\mathrm{P}}\left[b_{\mathrm{H}}-\left(\mu_{\mathrm{H}}+h_{\mathrm{H}}\right)\right]}{\mu_{\mathrm{H}}+h_{\mathrm{H}}}-\mu_{\mathrm{P}}
$$

or, again, piscivores are being fished to depletion or herbivores are being fished down to a population size that cannot support piscivores. In this case, herbivores will increase after reserve establishment if $h_{\mathrm{H}}>$ $h^{*}{ }_{\mathrm{H}, \text { inc,NSR, }}$ where:

$$
h_{\mathrm{H}, \mathrm{inc}, \mathrm{NSR}}^{*}=\frac{b_{\mathrm{P}} b_{\mathrm{H}}}{b_{\mathrm{P}}+\mu_{\mathrm{P}}}-\mu_{\mathrm{H}}
$$

or the effect of harvesting on herbivores before reserve establishment is greater than that of predation after reserve establishment. Note that $h^{*}{ }_{\mathrm{H}, \mathrm{inc}, \mathrm{SR}}<h^{*}{ }_{\mathrm{H} \text {,inc,NSR }}$ and $h^{*}{ }_{\mathrm{P}, \mathrm{dep}, \mathrm{SR}}<h^{*}{ }_{\mathrm{P}, \mathrm{dep}, \mathrm{NSR}}$.

Third, when $h_{\mathrm{P}}<h^{*}{ }_{\mathrm{P}, \mathrm{dep}, \mathrm{SR}}$, the opposite of the stability criterion for the equilibrium with no piscivores, the equilibrium with all 3 types exists and is locally stable. In this case, herbivores will increase after reserve establishment (i.e. the herbivore density in the equilibrium with all 3 types of species is greater with $h_{\mathrm{H}}=h_{\mathrm{P}}=0$ than with $h_{\mathrm{H}}$, $\left.h_{\mathrm{P}}>0\right)$ if $h_{\mathrm{P}}<h^{*}{ }_{\mathrm{P} \text {,inc, }}$ where:

$$
h_{\mathrm{P}, \text { inc }}^{*}=\frac{\gamma \mu_{\mathrm{P}} h_{\mathrm{H}}}{\mu_{\mathrm{H}}\left(\mu_{\mathrm{H}}+h_{\mathrm{H}}+\gamma\right)}
$$

or the increase in predation due to reduced piscivore harvest mortality after reserve establishment, depending on the herbivore growth rate into the larger size class, is less than the effect of harvest on herbivores before reserve establishment. Without size refugia $(\gamma=0)$, the equilibrium with all 3 types of species exists and is locally stable when $h_{\mathrm{P}}<h^{*}{ }_{\mathrm{P} \text {,dep,NSR, }}$ but in this case herbivores will always decrease after reserve establishment.

In summary, while piscivores always increase after reserve establishment (results not shown), whether herbivores increase or decrease depends on the piscivore and herbivore harvest mortalities before reserve establishment. Fig. 3 illustrates the relationship between herbivore increase and harvest mortalities using parameters from spotlight parrotfish Sparisoma viride for the herbivorous fish and Nassau grouper Epinephelus striatus for the piscivorous fish (Sadovy \& Eklund 1999, Choat et al. 2003, Muñoz \& Warner 2004, Mumby et al. 2006; Table A1 in Appendix 1), which are representative species from the empirical study that motivated this model (Mumby et al. 2006). Depending on harvest mortality before reserve establishment, the possible outcomes for the herbivores afterwards are:

(1) If, before reserve establishment, herbivores are fished at a rate that would eventually deplete the stock, herbivores will always increase after reserve establishment; this threshold is identical with and without prey size refugia (solid shaded region in Fig. 3).

(2) If, before reserve establishment, herbivores are not being fished to depletion but piscivores are (either due to fishing the piscivores to depletion or fishing the herbivores down to a population size that cannot support piscivores), herbivores increase after reserve establishment if the effect of harvest on herbivores before reserve establishment is greater than the effect of predation (horizontally and vertically striped re-

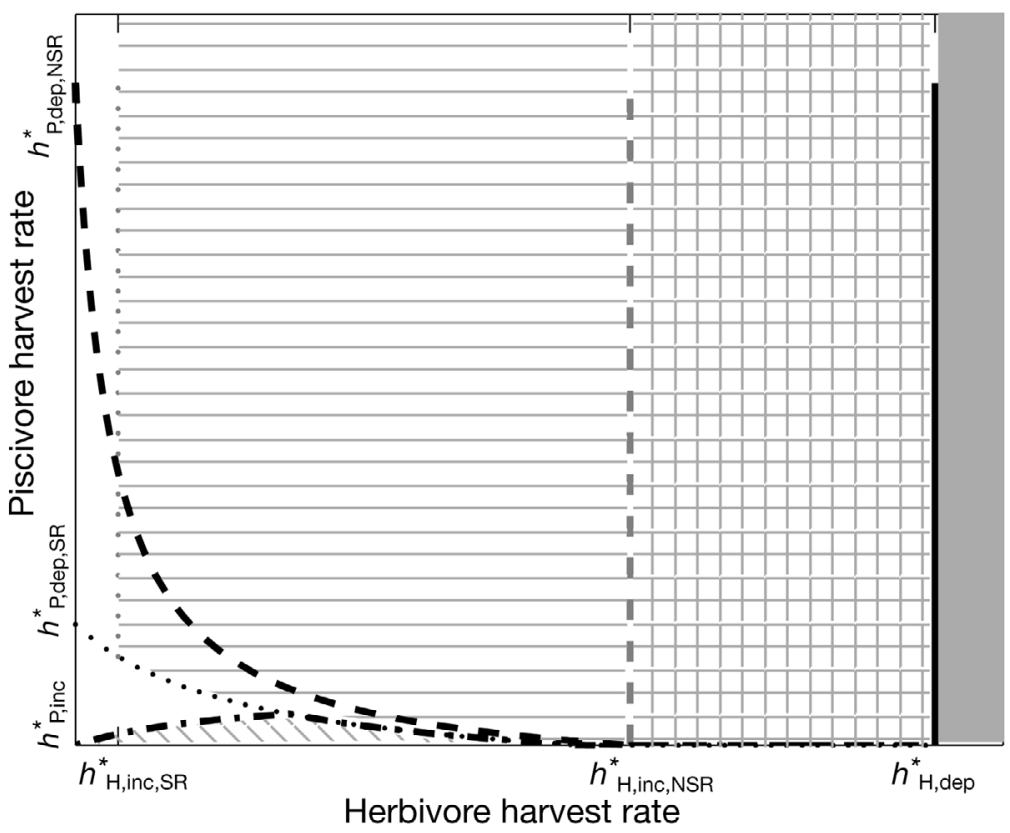

Fig. 3. Outcome of model dependent on harvest mortalities before reserve establishment; shaded and patterned regions indicate areas with expected increase in herbivores after reserve establishment (i.e. no cascading effects), depending on whether size refugia are accounted for. The black solid line is the threshold for fishing herbivores to depletion before reserve establishment (Eq. 7), and the black dotted and dashed lines for fishing piscivores to depletion with and without size refugia, respectively (Eqs. 8 \& 10). The grey dotted and dashed lines are the thresholds with and without size refugia, respectively, for an increase in herbivores when piscivores are being fished to depletion (Eqs. 9 \& 11). The black dash-dotted line is the threshold with size refugia for an increase in herbivores when neither piscivores or herbivores are being fished to depletion (Eq. 12). Given the parameter values in Table $\mathrm{A} 1, h^{*}{ }_{\mathrm{H}, \mathrm{dep}}=2.78 \mathrm{yr}^{-1}, h^{*}{ }_{\mathrm{H}, \text { inc,NSR }}=1.79 \mathrm{yr}^{-1}$, and $h^{*}{ }_{\mathrm{H}, \text { inc, SR }}=0.138 \mathrm{yr}^{-1} ;$ at $h_{\mathrm{H}}=$ $0, h_{\mathrm{P}, \mathrm{dep}, \mathrm{NSR}}^{*}=1.63 \mathrm{yr}^{-1}$ and $h_{\mathrm{P}, \mathrm{dep}, \mathrm{SR}}^{*}=0.297 \mathrm{yr}^{-1}$. Herbivores increase after reserve establishment when accounting for escapement size in the diagonally and horizontally striped regions, when ignoring escapement size in the vertically striped region, and in both cases in the solid shaded region 
gions for with and without size refugia, respectively, in Fig. 3). The threshold herbivore harvest mortality for this increase to occur is lower when accounting for size refugia; therefore, size refugia make an increase in herbivores after reserve establishment more likely when piscivores are being fished to depletion before reserve establishment.

(3) If neither herbivores or piscivores are being fished to depletion before reserve establishment, herbivores will always decrease after reserve establishment when ignoring size refugia. However, when accounting for size refugia, herbivores may increase if harvest on piscivores is below a threshold, such that the increase in predation after reserve establishment is less than the effect of harvest on herbivores before reserve establishment (diagonally striped region in Fig. 3). Therefore, size refugia make an increase in herbivores after reserve establishment more likely in this case.

\section{Including dynamics outside reserves}

Given the potential for openness in marine systems and thus the importance of dispersal dynamics to marine reserve design (Allison et al. 1998, Roberts et al. 2001), a particularly critical simplifying assumption made for analytic tractability in the above model is the exclusion of dynamics in harvested areas outside reserves. To determine the importance of this assumption, I numerically analyze the analogous model to Eqs. (1) to (4) with exchange between reserves and harvested areas. Detailed mathematically in Appendix 1, the model follows 2 pools of populations, in reserves and in harvested areas, for each type of species: resources, small herbivore, large herbivore, and piscivore populations. To model larval dispersal, exchange between the pools occurs in the reproduction/recruitment $(\beta)$ terms for herbivores and piscivores. The model ignores exchange in the resource populations because algae tend to have dispersal scales that are orders of magnitude smaller than for fish (Kinlan \& Gaines 2003). In numerical simulations I first integrate the model over time with harvesting in both pools until the populations reach an equilibrium and then integrate the model over time with no harvesting in the reserve populations. Simulation parameter values representative of Sparisoma viride and Epinephelus striatus are from the references listed above, with the addition of reserveharvested area exchange proportions estimated from Cowen et al. (2006) (Table A1).

In the numerical simulations without prey size refugia (Fig. 4, left hand column), trophic cascades of
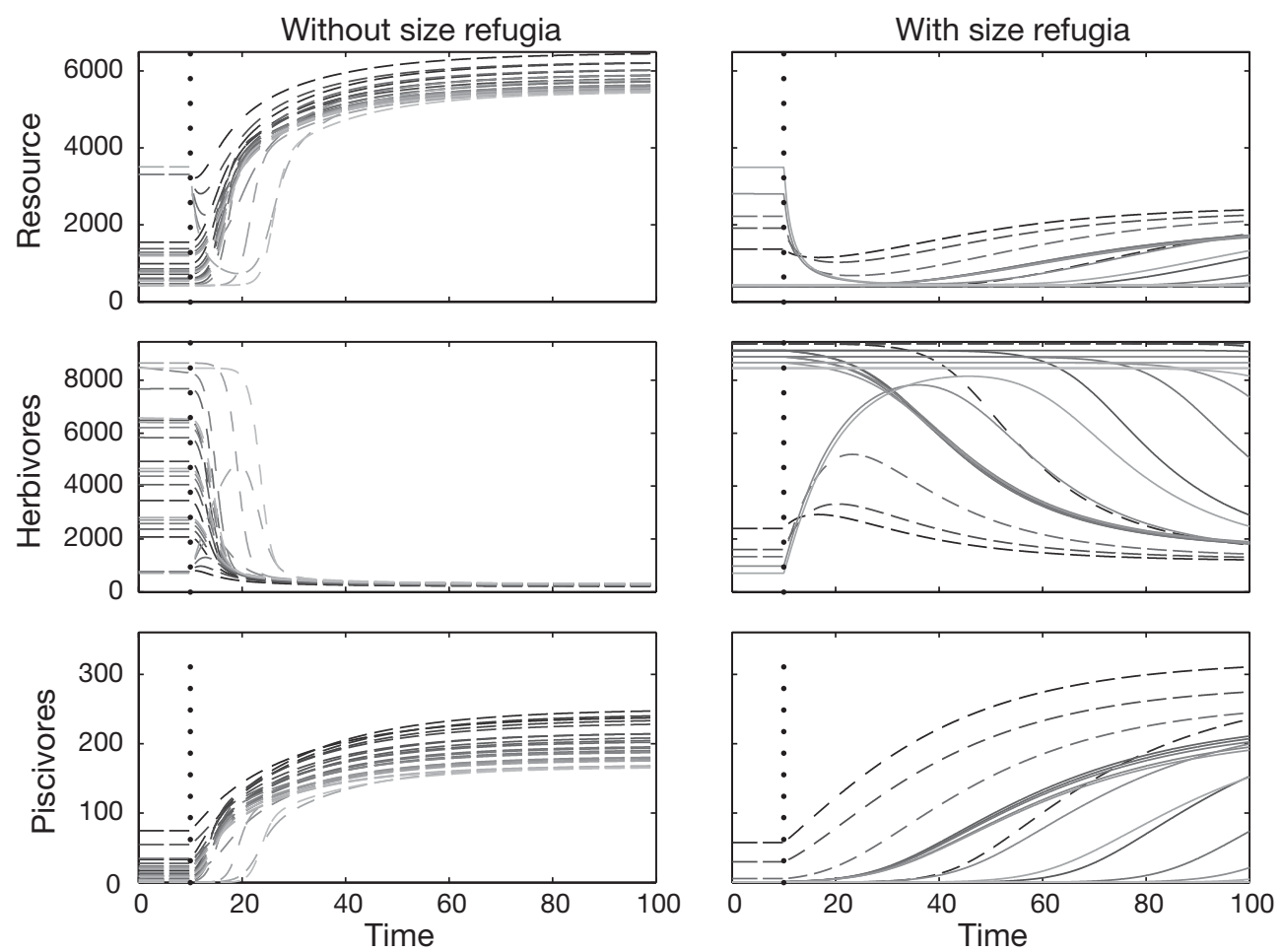

Fig. 4. Time trajectories of resource, herbivore, and piscivore populations within reserves for simulations of the model with exchange between reserves and harvested areas and with or without prey size refugia. Varying grayscale represents different simulations with varying herbivore and piscivore harvest rates before reserve establishment and outside reserves. Dashed and solid lines indicate cascades are and are not expected, respectively, from the analysis of the model without dynamics outside reserves

(Fig. 3). Vertical dotted lines indicate the time of reserve establishment 
increasing piscivores, decreasing herbivores, and increasing resources occur after reserve establishment given a wide array of harvest rates before establishment and outside reserves. While herbivore decreases and resource increases occur in some simulations with prey size refugia as well, constant population sizes or the opposite trends also occur, depending on the harvest rates (Fig. 4, right hand column). However, cascades do occur in some simulations with size refugia and harvest rates for which the basic model predicts an increase in herbivores. Therefore, the numerical simulations confirm that incorporating prey size refugia decreases the likelihood of trophic cascades in reserves when accounting for exchange with unprotected areas, but to a lesser extent than predicted when ignoring dynamics outside reserves.

\section{DISCUSSION}

Overall, prey size refugia make trophic cascades after reserve establishment less likely in the simple model and simulations presented here (Figs. $3 \& 4$ ). Trophic cascades do not occur with reserve establishment if the effect of harvest on herbivores before reserve establishment is greater than the effect of increased predation given piscivore release from harvest after reserve establishment. Incorporating prey size refugia, or adding a class of herbivores unavailable to and unaffected by piscivores, makes an increase in herbivores after reserve establishment more likely by reducing the effect of piscivores on herbivores.

While this model ignores biological realities such as greater reproduction, lower natural mortality, and greater harvest mortality before reserve establishment for the larger herbivores, these added complications all increase the contribution of larger herbivores after reserve establishment. Therefore, they would probably make the potential for size refugia to decrease the likelihood of trophic cascades more pronounced. However, some of the simplifying assumptions, such as resource-independent growth for the herbivores and ignoring increased piscivore size structure and therefore reduced herbivore size refugia after reserve establishment, may lead to an overestimation of the potential for size refugia to prevent trophic cascades. Further biological details, such as additional groups of interacting species and shifts in prey food source with size, may have unexpected and/or complex impacts on the model predictions and warrant future study.

The impact of size refugia on the expectation of species responses to reserve establishment in this simple model suggests that it is an important dynamic to consider when designing and monitoring marine reserves.
In addition to changing expectations for trophic cascades after reserve establishment, incorporating size refugia decreases the harvest rate threshold for fishing piscivores to depletion (Eqs. 8 \& 10); this increase in piscivore vulnerability to fishing with prey size refugia is due to smaller piscivore production with less prey available. Furthermore, the simulations with exchange between reserves and harvested areas indicate that piscivore recovery from fishing is slower when accounting for prey size refugia (Fig. 4). While these simulations ignore many dynamics, such as stochastic productivity and recruitment, important to predicting the time scale of species changes in abundances, this qualitative difference indicates the potential importance of prey size refugia for piscivore as well as herbivore response to reserve establishment.

With its simplistic representation of a marine community employed for analytic tractability, this model is not appropriate for quantitatively predicting community responses to reserve establishment. However, the large impact of size refugia on the model results suggests that incorporating size refugia may significantly change the predictions of more detailed and realistic simulations of specific marine communities developed for quantitative evaluation of management strategies. In addition, theoretical studies of marine systems with size-dependent predation in the absence of reserves suggest that the timing of predator and prey growth in response to productivity changes may be particularly important to theoretical predictions involving sizedependent predation (reviewed by Sogard 1997).

The theoretical potential for size refugia to prevent trophic cascades generalizes the empirical results of Mumby et al. (2006) to suggest that trophic cascades after reserve establishment are unlikely in systems where herbivores are harvested and have a size refugia from predation. The existence of prey size refugia is not unique to the parrotfish-grouper interactions in the Caribbean coral reef studied by Mumby et al. (2006). In both temperate and tropical aquatic ecosystems, empirical studies suggest that predation often decreases with increasing prey size for teleost fish preyed upon by fish and crustaceans; such size refugia can occur through predator gape size limitations or prey behavioral escapement tactics (reviewed by Sogard 1997). In addition to fish, prey size refugia exist in interactions between temperate intertidal invertebrates (e.g. Paine 1976, Paine \& Trimble 2004). Therefore, prey size refugia have a large potential to alter expectations after reserve establishment in many marine ecosystems.

Acknowledgements. Discussions with S. A. Levin and S. A. Kryazhimskiy were very helpful to the development of the research presented here. S. A. Levin, M. Urban, and 3 anony- 
mous reviewers provided insightful comments on an earlier version of this manuscript. Support for this research came from the National Science Foundation Graduate Research Fellowship Program and the Burroughs Wellcome Fund Training Program in Biological Dynamics (\#1001781).

\section{LITERATURE CITED}

Allison GW, Lubchenco J, Carr MH (1998) Marine reserves are necessary but not sufficient for marine conservation. Ecol Appl 8:S79-S92

Chase JM (1999) Food web effects of prey size refugia: variable interactions and alternative stable equilibria. Am Nat 154:559-570

Choat JH, Robertson DR, Ackerman JL, Posada JM (2003) An age-based demographic analysis of the Caribbean stoplight parrotfish Sparisoma viride. Mar Ecol Prog Ser 246: 265-277

Cowen RK, Paris CB, Srinivasan A (2006) Scaling of connectivity in marine populations. Science 311:522-527

Felippa CA, Park KC (2004) Synthesis tools for structural dynamics and partitioned analysis of coupled systems. In: Ibrahimbegovic A, Brank B (eds) Multi-physics and multi-scale computer models in non-linear analysis and optimal design of engineering structures under extreme conditions. NATO ARW PST. ARW 980262, University of Ljubliana, p 50-110

Halpern BS (2003) The impact of marine reserves: Do reserves work and does reserve size matter? Ecol Appl 13:S117-S137

Kinlan BP, Gaines SD (2003) Propagule dispersal in marine and terrestrial environments: A community perspective. Ecology 84:2007-2020

Mumby PJ (2006) The impact of exploiting grazers (Scaridae) on the dynamics of Caribbean coral reefs. Ecol Appl $16: 747-769$
Mumby PJ, Dahlgren CP, Harborne AR, Kappel CV and 10 others (2006) Fishing, trophic cascades, and the process of grazing on coral reefs. Science 311:98-101

Muñoz RC, Warner RR (2004) Testing a new version of the size-advantage hypothesis for sex change: sperm competition and size-skew effects in the bucktooth parrotfish, Sparisoma radians. Behav Ecol 15:129-136

Paine RT (1976) Size-limited predation: an observational and experimental approach with the Mytilus-Pisaster interaction. Ecology 57:858-873

Paine RT, Trimble AC (2004) Abrupt community change on a rocky shore-biological mechanisms contributing to the potential formation of an alternative state. Ecol Lett 7: 441-445

Pauly D, Christensen V, Dalsgaard J, Froese R, Torres F Jr (1998) Fishing down marine food webs. Science 279: 860-863

Pinnegar JK, Polunin NVC, Francour P, Badalamenti F and 7 others (2000) Trophic cascades in benthic marine ecosystems: Lessons for fisheries and protected-area management. Environ Conserv 27:179-200

Polis GA, Strong DR (1996) Food web complexity and community dynamics. Am Nat 147:813-846

Roberts CM, Halpern B, Palumbi SR, Warner, RR (2001) Designing marine reserve networks: why small, isolated protected areas are not enough. Conserv Biol Pract 2:10-17

Sadovy Y, Eklund A (1999) Synopsis of biological data on the Nassau grouper, Epinephelus striatus (Bloch, 1792), and the jewfish, E. itajara (Lichtenstein, 1822). Tech Rep NMFS-146, NOAA, Seattle, WA

Sogard SM (1997) Size-selective mortality in the juvenile stage of teleost fishes: a review. Bull Mar Sci 60: 1129-1157

Walters C (2000) Impacts of dispersal, ecological interactions, and fishing effort dynamics on efficacy of marine protected areas: How large should protected areas be? Bull Mar Sci 66:745-757 
Appendix 1. Mathematical details of the model analysis and simulations

\section{Local stability analysis}

In order to simplify the analysis of the model with prey size refugia, the model was non-dimensionalized by substituting:

$$
\begin{array}{rl}
x=R / R_{\mathrm{IN}} & U_{\mathrm{H}}=\left(\mu_{\mathrm{H}}+h_{\mathrm{H}}\right) / D \\
y_{1}=\delta_{\mathrm{H}} H_{\mathrm{S}} / D & U_{\mathrm{P}}=\left(\mu_{\mathrm{P}}+h_{\mathrm{P}}\right) / D \\
y_{2}=\delta_{\mathrm{H}} H_{\mathrm{L}} / D & B_{\mathrm{H}}=R_{\mathrm{IN}} \delta_{\mathrm{H}} \beta_{\mathrm{H}} / D \\
z=\delta_{\mathrm{P}} P / D & B_{\mathrm{P}}=\beta_{\mathrm{P}} \delta_{\mathrm{P}} / \delta_{\mathrm{H}} \\
\tau=D t & G=\gamma / D
\end{array}
$$

The equivalent to Eqs. (1) to (4) with the above dimensionless variables and parameters is:

$$
\begin{aligned}
& \frac{\mathrm{d} x}{\mathrm{~d} \tau}=(1-x)-\left(y_{1}+y_{2}\right) x \\
& \frac{\mathrm{d} y_{1}}{\mathrm{~d} \tau}=B_{\mathrm{H}}\left(y_{1}+y_{2}\right) x-\left(G+U_{\mathrm{H}}+z\right) y_{1} \\
& \frac{\mathrm{d} y_{2}}{\mathrm{~d} \tau}=G y_{1}-U_{\mathrm{H}} y_{2} \\
& \frac{\mathrm{d} z}{\mathrm{~d} \tau}=\left(B_{\mathrm{P}} y_{1}-U_{\mathrm{P}}\right) z
\end{aligned}
$$

To determine whether the herbivores increase after reserve establishment, first I determine the expected population densities before reserve establishment, depending on harvest mortalities. Assuming species have been harvested for a long enough period such that their densities are approaching equilibrium, the expected population densities are the locally stable equilibrium population densities for a given set of parameters. There are 3 possible equilibria in the model: one with the resource only and no herbivores or piscivores, one with the resource and herbivores but no piscivores, and one with all 3 types of species.

The equilibrium with the resource only is:

$$
\begin{aligned}
& \bar{x}=1 \\
& \bar{y}_{1}=0 \\
& \bar{y}_{2}=0 \\
& \bar{z}=0
\end{aligned}
$$

The Jacobian of Eqs. (13) to (16) evaluated at the above equilibrium is:

$$
\left(\begin{array}{cccc}
-1 & -1 & -1 & 0 \\
0 & B_{\mathrm{H}}-\left(U_{\mathrm{H}}+G\right) & B_{\mathrm{H}} & 0 \\
0 & G & -U_{\mathrm{H}} & 0 \\
0 & 0 & 0 & -U_{\mathrm{P}}
\end{array}\right)
$$

The eigenvalues of the above matrix are $-1,-U_{\mathrm{P}}$ and the eigenvalues of the center $2 \times 2$ matrix; thus the eigenvalues for the center $2 \times 2$ matrix are the only ones with the potential to be positive. The Routh-Hurwitz criteria for negative eigenvalues and therefore local stability for the central $2 \times 2$ matrix (trace $<0$, determinant $>0$ ) simplify to $U_{\mathrm{H}}>B_{\mathrm{H}}$. Substituting in terms of the original (dimensional) parameters, the equilibrium with the resource only is locally stable when $h_{\mathrm{H}}>h^{*}{ }_{\mathrm{H}, \mathrm{dep}}$ where $h^{*}{ }_{\mathrm{H} \text {,dep }}$ is defined in Eq. (7). In this case, the expected herbivore density $\left(\bar{y}_{1}+\bar{y}_{2}\right)$ before reserve establishment is:

$$
\bar{y}_{X}=0
$$

The stability criteria and expected herbivore density are independent of $G$; therefore, they are identical with and without size refugia.

The equilibrium with the resource and herbivores but no piscivores is:

$$
\begin{aligned}
& \bar{x}=\frac{U_{\mathrm{H}}}{B_{\mathrm{H}}} \\
& \bar{y}_{1}=\frac{B_{\mathrm{H}}-U_{\mathrm{H}}}{U_{\mathrm{H}}+G} \\
& \bar{y}_{2}=\frac{G}{U_{\mathrm{H}}}\left(\frac{B_{\mathrm{H}}-U_{\mathrm{H}}}{U_{\mathrm{H}}+G}\right) \\
& \bar{z}=0
\end{aligned}
$$

$B_{\mathrm{H}}>U_{\mathrm{H}}$ (equivalent to $h^{*}{ }_{\mathrm{H}}<h_{\mathrm{H}, \mathrm{dep}}$ ) is necessary for the equilibrium to exist biologically $\left(\bar{y}_{1}, \bar{y}_{2}>0\right)$. The Jacobian of Eqs. (13) to (16) evaluated at the above equilibrium is:

$$
\left(\begin{array}{cccc}
-\frac{B_{\mathrm{H}}}{U_{\mathrm{H}}} & -\frac{U_{\mathrm{H}}}{B_{\mathrm{H}}} & -\frac{U_{\mathrm{H}}}{B_{\mathrm{H}}} & 0 \\
\frac{B_{\mathrm{H}}}{U_{\mathrm{H}}}\left(B_{\mathrm{H}}-U_{\mathrm{H}}\right) & -G & U_{\mathrm{H}} & -\frac{B_{\mathrm{H}}-U_{\mathrm{H}}}{U_{\mathrm{H}}+G} \\
0 & G & -U_{\mathrm{H}} & 0 \\
0 & 0 & 0 & \frac{B_{\mathrm{P}}\left(B_{\mathrm{H}}-U_{\mathrm{H}}\right)}{U_{\mathrm{H}}+G}-U_{\mathrm{P}}
\end{array}\right)
$$

The eigenvalues of the above matrix are $B_{\mathrm{P}}\left(B_{\mathrm{H}}-U_{\mathrm{H}}\right) /\left(U_{\mathrm{H}}\right.$ $+G)-U_{\mathrm{P}}$ and the eigenvalues of the upper left $3 \times 3$ matrix. The upper left $3 \times 3$ matrix satisfies the Routh-Hurwitz criteria for negative eigenvalues for a $3 \times 3$ matrix (trace $<0$, determinant $<0$, and trace $\times$ sum of the principal minor $2 \times$ 2 determinants $<$ determinant) given $B_{\mathrm{H}}>U_{\mathrm{H}}$. Therefore, the equilibrium without piscivores is locally stable when $U_{\mathrm{p}}>B_{\mathrm{P}}\left(B_{\mathrm{H}}-U_{\mathrm{H}}\right) /\left(U_{\mathrm{H}}+G\right)$, or, in terms of the original parameters, $h_{\mathrm{p}}>h^{*}{ }_{\mathrm{P}, \mathrm{dep}, \mathrm{SR}}$, where $h^{*}{ }_{\mathrm{P}, \mathrm{dep}, \mathrm{SR}}$ is defined in Eq. (8). In this case, the expected herbivore population $\left(\bar{y}_{1}+\bar{y}_{2}\right)$ before reserve establishment is:

$$
\bar{y}_{\mathrm{xy}, \mathrm{SR}}=\frac{B_{\mathrm{H}}-U_{\mathrm{H}}}{U_{\mathrm{H}}}
$$

Without size refugia $(G=0)$, the equilibrium is locally stable when $h_{\mathrm{p}}>h^{*}{ }_{\mathrm{P}, \mathrm{dep}, \mathrm{NSR}}$, where $h_{\mathrm{P}, \mathrm{dep}, \mathrm{NSR}}$ is defined in Eq. (10), and the expected herbivore population before reserve establishment is the same as with size refugia (Eq. 28).

The equilibrium with all 3 types of species is:

$$
\begin{aligned}
& \bar{x}=\frac{B_{\mathrm{P}} U_{\mathrm{H}}}{B_{\mathrm{P}} U_{\mathrm{H}}+U_{\mathrm{P}} G+U_{\mathrm{P}} U_{\mathrm{H}}} \\
& \bar{y}_{1}=\frac{U_{\mathrm{P}}}{B_{\mathrm{P}}} \\
& \bar{y}_{2}=\frac{G U_{\mathrm{P}}}{U_{\mathrm{H}} B_{\mathrm{P}}} \\
& \bar{z}=\frac{G+U_{\mathrm{H}}}{B_{\mathrm{P}} U_{\mathrm{H}}+U_{\mathrm{P}} G+U_{\mathrm{P}} U_{\mathrm{H}}}\left[B_{\mathrm{P}} B_{\mathrm{H}}-\left(B_{\mathrm{P}} U_{\mathrm{H}}+U_{\mathrm{P}} G+U_{\mathrm{P}} U_{\mathrm{H}}\right)\right]
\end{aligned}
$$

The Jacobian of Eqs. (13) to (16) evaluated at the above equilibrium is:

$$
\left(\begin{array}{cccc}
-\frac{1}{\bar{x}} & -\bar{x} & -\bar{x} & 0 \\
\frac{B_{\mathrm{H}} U_{\mathrm{P}}}{B_{\mathrm{P}} U_{\mathrm{H}}}\left(U_{\mathrm{H}}+G\right) & -\frac{G B_{\mathrm{H}} \bar{x}}{U_{\mathrm{H}}} & B_{\mathrm{H}} \bar{x} & -\frac{U_{\mathrm{P}}}{B_{\mathrm{P}}} \\
0 & G & -U_{\mathrm{H}} & 0 \\
0 & B_{\mathrm{P}} \bar{z} & 0 & 0
\end{array}\right)
$$

The above matrix satisfies the Routh-Hurwitz criteria for negative eigenvalues for a $4 \times 4$ matrix (trace $<0$, determinant $>0$, trace $\times$ determinant $>$ sum of the principal minor $3 \times 3$ determinants $\times$ the sum of the principal minor $2 \times 2$ determinants, and sum of the principal minor $2 \times 2$ determi- 
Appendix 1 (continued)

nants $\times$ sum of the principal minor $3 \times 3$ determinants $\times$ trace $>$ determinant $\times$ trace $^{2}+$ sum of the principal minor $3 \times 3$ determinants $^{2}$; Felippa \& Park 2004) when the equilibrium exists biologically $\left(\bar{z}>0\right.$, or $U_{\mathrm{p}}<B_{\mathrm{P}}\left(B_{\mathrm{H}}-U_{\mathrm{H}}\right) /\left(U_{\mathrm{H}}+G\right)$, which is equivalent to $\left.h_{\mathrm{p}}<h^{*}{ }_{\mathrm{P}, \mathrm{dep}, \mathrm{SR}}\right)$. Therefore, the equilibrium with all 3 types of species is locally stable when $h_{\mathrm{p}}<$ $h^{*}{ }_{\mathrm{P}, \mathrm{dep}, \mathrm{SR}}$. In this case, the expected herbivore population $\left(\bar{y}_{1}+\bar{y}_{2}\right)$ before reserve establishment is

$$
\bar{y}_{\mathrm{xyz}, \mathrm{SR}}=\frac{U_{\mathrm{P}}\left(U_{\mathrm{H}}+G\right)}{B_{\mathrm{P}} U_{\mathrm{H}}}
$$

Without size refugia $(G=0)$ the equilibrium is locally stable when $h_{\mathrm{p}}<h_{\mathrm{P}, \mathrm{dep}, \mathrm{NSR} \text {, }}^{*}$ and the expected herbivore population before reserve establishment is

$$
\bar{y}_{\mathrm{xyz}, \mathrm{NSR}}=\frac{U_{\mathrm{P}}}{B_{\mathrm{P}}}
$$

After reserve establishment $\left(h_{\mathrm{H}}, h_{\mathrm{P}} \rightarrow 0\right.$, or $U_{\mathrm{H}}:\left(\mu_{\mathrm{H}}+\right.$ $\left.h_{\mathrm{H}}\right) / D \rightarrow \mu_{\mathrm{H}} / D$ and $\left.U_{\mathrm{P}}:\left(\mu_{\mathrm{P}}+h_{\mathrm{P}}\right) / D \rightarrow \mu_{\mathrm{P}} / D\right)$, I assume the parameter values are such that the equilibrium with all 3 types of species exists biologically and is locally stable $\left(\mu_{\mathrm{H}}<\beta_{\mathrm{H}} \delta_{\mathrm{H}} R_{\mathrm{IN}}\right.$ and $\mu_{\mathrm{p}}<\frac{\beta_{\mathrm{P}} \delta_{\mathrm{P}} D}{\delta_{\mathrm{H}}\left(\mu_{\mathrm{H}}+\gamma\right)}\left(R_{\mathrm{IN}} \beta_{\mathrm{H}} \delta_{\mathrm{H}}-\mu_{\mathrm{H}}\right)$, or reproduction outweighs mortality for herbivores and piscivores). Therefore, the expected herbivore population after reserve establishment, given enough time for recovery from fishing, is $\bar{y}_{\mathrm{Xyz}, \mathrm{SR}}$ from Eq. (34) with size refugia or $\bar{y}_{\mathrm{xyz}, \mathrm{NSR}}$ from Eq. (35) without size refugia, where $U_{\mathrm{H}}=\mu_{\mathrm{H}} / D$ and $U_{\mathrm{p}}=$ $\mu_{\mathrm{P}} / D$. Comparing these values to the expected herbivore population before reserve establishment (Eqs. 22, 28 \& 34 with size refugia or Eq. 35 without size refugia, where $U_{\mathrm{H}}=$ $\left(\mu_{\mathrm{H}}+h_{\mathrm{H}}\right) / D$ and $\left.U_{\mathrm{p}}=\left(\mu_{\mathrm{P}}+h_{\mathrm{P}}\right) / D\right)$ indicates whether the herbivores will increase or decrease after reserve establishment, depending on the harvest mortalities before reserve establishment.

\section{Model extension with dynamics outside reserves}

Below are the dynamics of the model with exchange between reserves and harvested areas. Let the subscripts $r$ and $\mathrm{h}$ indicate reserve and harvested area, respectively, for the resource, herbivore, and piscivore populations. In addition, let $m_{\mathrm{N}, \mathrm{rh}}$ be the proportion of larval that move from reserve(s) to harvested areas and $m_{\mathrm{N}, \mathrm{hr}}$ be the proportion of larval that move from harvested areas to reserve(s) for species $N$ (herbivores $H$ or piscivores $P$ ). Then the dynamics are:

$$
\begin{aligned}
& \frac{\mathrm{d} R_{\mathrm{h}}}{\mathrm{d} t}= D\left(R_{\mathrm{IN}}-R_{\mathrm{h}}\right)-\delta_{\mathrm{H}}\left(H_{\mathrm{Sh}}+H_{\mathrm{Lh}}\right) R_{\mathrm{h}} \\
& \frac{\mathrm{d} H_{\mathrm{Sh}}}{\mathrm{d} t}=\begin{array}{c}
\left(1-m_{\mathrm{H}, \mathrm{hr}}\right) \beta_{\mathrm{H}} \delta_{\mathrm{H}}\left(H_{\mathrm{Sh}}+H_{\mathrm{Lh}}\right) R_{\mathrm{h}}- \\
\left(\gamma+\mu_{\mathrm{H}}+h_{\mathrm{H}}+\delta_{\mathrm{P}} P_{\mathrm{h}}\right) H_{\mathrm{Sh}}+m_{\mathrm{H}, \mathrm{rh}} \beta_{\mathrm{H}} \delta_{\mathrm{H}}\left(H_{\mathrm{Sr}}+H_{\mathrm{Lr}}\right) R_{\mathrm{r}}
\end{array} \\
& \frac{\mathrm{d} H_{\mathrm{Lh}}}{\mathrm{d} t}=\gamma H_{\mathrm{Sh}}-\left(\mu_{\mathrm{H}}+h_{\mathrm{H}}\right) H_{\mathrm{Lh}} \\
& \frac{\mathrm{d} P_{\mathrm{h}}}{\mathrm{d} t}=\left[\left(1-m_{\mathrm{P}, \mathrm{hr}}\right) \beta_{\mathrm{P}} \delta_{\mathrm{P}} H_{\mathrm{Sh}}-\left(\mu_{\mathrm{P}}+h_{\mathrm{P}}\right)\right] P_{\mathrm{h}}+m_{\mathrm{P}, \mathrm{rh}} \beta_{\mathrm{P}} \delta_{\mathrm{P}} H_{\mathrm{Sr}} P_{\mathrm{r}} \\
& \frac{\mathrm{d} R_{\mathrm{r}}}{\mathrm{d} t}=D\left(R_{\mathrm{IN}}-R_{\mathrm{r}}\right)-\delta_{\mathrm{H}}\left(H_{\mathrm{Sr}}+H_{\mathrm{Lr}}\right) R_{\mathrm{r}} \\
& \frac{\mathrm{d} H_{\mathrm{Sr}}}{\mathrm{d} t}=\left(1-m_{\mathrm{H}, \mathrm{rh}}\right) \beta_{\mathrm{H}} \delta_{\mathrm{H}}\left(H_{\mathrm{Sr}}+H_{\mathrm{Lr}}\right) R_{\mathrm{r}}-\left(\gamma+\mu_{\mathrm{H}}+\delta_{\mathrm{P}} P_{\mathrm{r}}\right) H_{\mathrm{Sr}}+ \\
& \quad m_{\mathrm{H}, \mathrm{hr}} \beta_{\mathrm{H}} \delta_{\mathrm{H}}\left(H_{\mathrm{Sh}}+H_{\mathrm{Lh}}\right) R_{\mathrm{h}} \\
& \frac{\mathrm{d} H_{\mathrm{Lr}}}{\mathrm{d} t}=\gamma H_{\mathrm{Sr}}-\mu_{\mathrm{H}} H_{\mathrm{Lr}} \\
& \frac{\mathrm{d} P_{\mathrm{r}}}{\mathrm{d} t}=\left[\left(1-m_{\mathrm{P}, \mathrm{rh}}\right) \beta_{\mathrm{P}} \delta_{\mathrm{P}} H_{\mathrm{Sr}}-\mu_{\mathrm{P}}\right] P_{\mathrm{r}}+m_{\mathrm{P}, \mathrm{hr}} \beta_{\mathrm{P}} \delta_{\mathrm{P}} H_{\mathrm{Sh}} P_{\mathrm{h}}
\end{aligned}
$$

where all other parameters are as in Eqs. (1) to (4). Also as above, letting $\gamma=0$ yields the analogous model without prey size refugia.

Table A1 provides parameter values both for the results presented in Fig. 3 and for the numerical simulations of Eqs. (36) to (43) presented in Fig. 4. Note that some parameter values are calculated, rather than directly taken, from the references. In particular: (1) reproductive efficiencies are $10 \%$ of the gonadosomatic indices in the references to account for energy lost during predation; (2) natural mortality rates are the inverse of the average lifespans; (3) growth rate from the small to large herbivore size class is the inverse of the expected age at which herbivores reach size refugia, based on von Bertalanffy growth parameters; (4) movement proportions depend on the reported selfrecruitment percent such that non-self-recruiting larvae are equally likely to settle in reserves or in harvested areas.

Table A1. Parameter values used in Figs. 3 \& 4 based on Sparisoma viride and Epinephelus striatus for herbivores and piscivores, respectively. $R, H$ and $P$ indicate units of resource, herbivore and piscivore population size, respectively. For movement and harvest parameters, $N$ indicates any species (herbivores $H$ or piscivores $P$ ) and $x$ and $y$ indicate location: reserve $\mathrm{r}$ or harvested area $h$

\begin{tabular}{|llll|}
\hline Description & Parameter & \multicolumn{1}{c|}{ Value(s) } & Reference(s) \\
\hline Resource input & $R_{\mathrm{IN}}$ & $10000 R$ & \\
Resource flow rate & $D$ & $10 \mathrm{yr}^{-1}$ & \\
Herbivore predation rate & $\delta_{\mathrm{H}}$ & $0.0263 \mathrm{H}^{-1} \mathrm{yr}^{-1}$ & Mumby et al. (2006) \\
Piscivore predation rate & $\delta_{\mathrm{P}}$ & $0.01 \mathrm{P}^{-1} \mathrm{yr}^{-1}$ & \\
Herbivore reproductive efficiency & $\beta_{\mathrm{H}}$ & $0.011 \mathrm{HR}^{-1}$ & Muñoz \& Warner (2004) \\
Piscivore reproductive efficiency & $\beta_{\mathrm{P}}$ & $0.0175 \mathrm{PH}^{-1}$ & Sadovy \& Eklund (1999) \\
Herbivore natural mortality & $\mu_{\mathrm{H}}$ & $0.111 \mathrm{yr}^{-1}$ & Choat et al. (2003) \\
Piscivore natural mortality & $\mu_{\mathrm{P}}$ & $0.0345 \mathrm{yr}^{-1}$ & Sadovy \& Eklund (1999) \\
Herbivore growth rate & $\gamma$ & $0.447 \mathrm{yr}^{-1}$ & Choat et al. (2003), Mumby et al. (2006) \\
Movement proportions & $m_{\mathrm{N}, \mathrm{xy}}$ & 0.393 & Cowen et al. (2006) \\
Harvest mortalities & $h_{\mathrm{N}}$ & $0.1,0.3,0.5,0.7,0.9 \mathrm{yr}^{-1}$ & \\
\hline
\end{tabular}

